Bond University

Research Repository

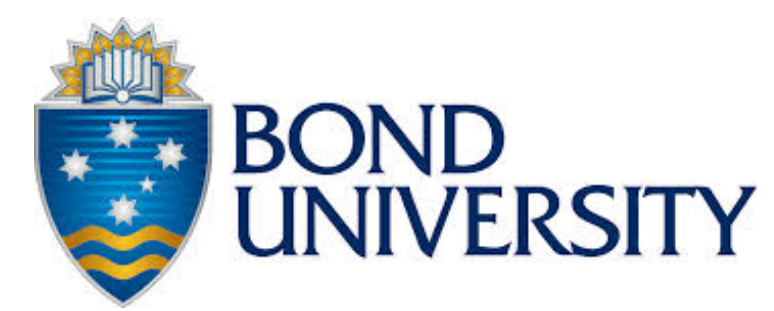

\title{
Early warning biomarkers in major depressive disorder: a strategic approach to a testing question
}

Pratt, Robin; Stapelberg, N J C

Published in:

Biomarkers

DOI:

10.1080/1354750X.2018.1463563

Licence:

Unspecified

Link to output in Bond University research repository.

Recommended citation(APA):

Pratt, R., \& Stapelberg, N. J. C. (2018). Early warning biomarkers in major depressive disorder: a strategic approach to a testing question. Biomarkers, 23(6), 563-572. https://doi.org/10.1080/1354750X.2018.1463563

\section{General rights}

Copyright and moral rights for the publications made accessible in the public portal are retained by the authors and/or other copyright owners and it is a condition of accessing publications that users recognise and abide by the legal requirements associated with these rights.

For more information, or if you believe that this document breaches copyright, please contact the Bond University research repository coordinator. 
Early warning biomarkers in major depressive disorder: a strategic

\section{approach to a testing question}

\section{R.Pratt, N.J.C. Stapelberg}

Dr Robin Pratt (corresponding author)

\section{MB ChB MBA AMInstD FRCPA FRANZCP}

Consultation Liaison Psychiatry, Nepean Hospital, Penrith, NSW 2751, Australia

E-mail: robin.pratt@health.nsw.gov.au

Professor Nicolas J.C. Stapelberg

BSc (Hons), MBBS, PhD, FRANZCP

Faculty of Health Sciences and Medicine, Bond University and Gold Coast Hospital and Health Service, 1 Hospital Boulevard, Southport, Queensland 4215, Australia 


\title{
Early warning biomarkers in major depressive disorder: a strategic approach to a testing question
}

\author{
Abstract \\ Purpose \\ Identification of biomarkers in Major Depressive Disorder (MDD) has proceeded in an \\ extemporized manner. No single biomarker has been identified with utility in screening, \\ diagnosis, prognosis or monitoring, and screening tests have different characteristics \\ than the other functions. Using Chaos, Bifurcation and Perturbation (CBP) Theories, \\ the aim is to identify biomarkers to aid clinicians in screening for MDD.

\section{Materials and methods} \\ MDD is a complex disorder; consequently, a reductionist approach to characterize the \\ complex system changes found in MDD will be inchoate and unreliable. A holistic \\ approach is used to identify biomarkers reflecting the tipping points seen before the \\ catastrophic bifurcation that results in MDD.
}

\section{Results}

Applying CBP Theories revealed skew, resistance to change, flickering, increased variance and autocorrelation as patterns of biomarkers. Integrals and differentials of extracellular and intracellular biomarkers were identified, specifically focussed on hypothalamo-pituitary axis (HPA) axis dysfunction, metabolic dysfunction, inflammation and mitochondrial oxidative stress, and tryptophan metabolism.

\section{Conclusion}

Applying CBP Theories to the dysfunctional complex biological systems in MDD led to development of integrals and differentials of biomarkers that can be used in screening for MDD and planning future biomarker research, targeting intracellular and extracellular inflammation, HPA axis dysfunction, and tryptophan metabolism. 
Key Words: Bifurcation Theory, Chaos Theory, biomarkers, depression, major depressive disorder, depression screening, Perturbation Theory.

\section{Introduction}

Major Depressive Disorder (MDD) causes significant functional impairment (Kessler $e t$ al., 2003), incurs significant public health costs (Kessler, 2012), and contributes to approximately $10 \%$ of the global burden of disease (World Health Organisation, 2009).

Moreover, there is evidence of an increase in the rate of MDD (Ferrari et al, 2012). The US Preventive Services Task Force has recommended that primary care providers should screen all adults for MDD (Siu et al., 2016).

Biomarker research has focused on the development of a single biomarker or small group of biomarkers that can be used to diagnose, rather than screen for, MDD (Boksa, 2013, Ebot Enaw and Smith, 2013, Bilello et al., 2015, Kunugi et al., 2015, De Long, 2016, Young et al., 2016). Commentary in the American Association of Clinical Chemistry (De Long, 2016) and others (Kunugi et al., 2015) suggest that the reductionist approach of searching for a single test for MDD has been unrewarding. Twelve recent reviews from 2011 to 2017 , including those above, identified different arrays of biomarkers with only minimal overlap in core biomarkers between reviews (Papakostas et al., 2011, Lang and Borgwardt, 2013, Lopresti et al., 2014, Seib et al., 2014, Zheng et al., 2016, Harada et al., 2017, Machado-Vieira et al., 2017, Strawbridge et al., 2017). Recent advances have considered a group of tests, forming a profile of biomarkers such as the MDDScore, rather than a single test (Bilello et al., 2015).

MDD is a complex biological disorder where multiple biological systems have been shown to be dysfunctional (Stapelberg et al., 2015). It is proposed that a strategic 
and holistic approach to the development of biomarkers for MDD is required. In addition, differing biomarkers for screening, diagnosis, monitoring and prognosis in MDD are required because the characteristics of tests required fulfilling these functions are significantly different.

Tipping points occur before catastrophic bifurcations (Scheffer et al., 2009) such as the transition from health to MDD and involve dysfunction of the HPA axis (Sapolsky, 2000), metabolic state (Winokur et al., 1988), tryptophan metabolism (Ruddick et al., 2006), inflammatory control and mitochondrial oxidative stress (Khanzode et al., 2003). Applying Chaos, Bifurcation and Perturbation Theories to these dysfunctional biological systems facilitates development of a profile of biomarkers to screen high prevalence groups for MDD. As the systems and the changes within them are so complex, it is proposed that biomarkers which are measured as absolute levels, integrals and differentials will show the skew, resistance to change, flickering, increased variance, and autocorrelation seen in tipping points prior to catastrophic bifurcation, and will be significantly more valuable than a single biomarker measured at a single point in time.

\section{Biomarkers in MDD}

MDD is currently defined using a categorical classification system. DSM 5 (American Psychiatric Association, 2013) and ICD 10 (World Health Organization, 1992), both delineate diagnostic criteria for MDD that are based on subjective patient assessments and structured, but subjective, clinician assessments. There have been attempts to introduce objective testing to aid in diagnosis, screening, prognosis, or monitoring of treatment response for psychiatric disorders. For example, the inclusion of cerebrospinal fluid hypocretin-1 (orexin-A) testing into the diagnostic criteria for 
narcolepsy represents the first use of a laboratory test to support a formal psychiatric diagnosis (criterion (B2), p373 in DSM 5 (American Psychiatric Association, 2013)).

An alternative approach has been to use multiple biomarkers together as a single investigative entity (Papakostas et al., 2011), such as the MDDScore test (Bilello et al., 2015). Sensitivity and specificity of the MDDScore test have been reported to be as high as $92 \%$ and $81 \%$ respectively.

Using an holistic approach, the intent in this paper is to identify from the literature by applying the methodology delineated, an initial group of analytes that could be used as biomarkers of the tipping points that are precursors to the clinical depressive state.

\section{Clinical Significance}

- Strategic methodology for the development of biomarkers in MDD

- Developing biomarkers for aiding in screening high risk populations for MDD

- Developing biomarkers for aiding in diagnosis of MDD

- Developing prognostic biomarkers for MDD

\section{Materials and Methods}

\section{Functions of Tests or Biomarkers}

Tests of individual biomarkers are undertaken for one of four main reasons (Mayeux, 2004, Pariante and Lightman, 2008a, Burtis et al., 2015a):

- $\quad$ Screening

- Diagnosis, including identification of subtypes

- Monitoring disorder progress or treatment response

- Prognosis 
As the characteristics of each are inherently different, this initial research is restricted to screening for MDD targeting the tipping point transformations that occur prior to catastrophic bifurcations.

\section{Application of foundation theories}

Perturbation Theory is a method for finding an approximate solution to a complex problem by starting from the exact solution of a related, simpler problem. A critical feature of the technique is a middle step that breaks the problem into "solvable" and "perturbation" parts (Bogolyubov, 2011). Perturbation Theory was applied to the problem of developing biomarkers in MDD. In a myocardial infarction, changes in a single test such as troponin I can be used in initial screening of high risk patients with chest pain, and, after a repeat test, diagnose myocardial infarction. The change over time is the diagnostic criterion. From this solvable part, the concept of interpolating the variable of time into biomarker measurement in MDD gave rise to the perturbations suggesting value in integrals and differentials of biomarker measurements in the complex disorder that is MDD.

Chaos Theory describes dynamical systems that evolve over time. These complex systems are highly sensitive to initial conditions in a deterministic way, but can have major perturbations over the long term, which, as described by the butterfly effect, can lead to catastrophic bifurcations and result in new stable pathological states (Ott, 2002, Boeing, 2016). Feedback often becomes chaotic prior to critical transition to the new stable, but pathological, state. The state prior to the catastrophic bifurcation is a tipping point and may herald development of the new pathological state. Identifying tipping points could serve as potential indicators for the development of MDD. 
Bifurcation Theory is most commonly applied to the study of dynamical systems and is concerned with the sudden changes that occur in a system when one or more parameters are varied (Arnold and Novikov, 1994). When combined with Chaos Theory, it is proposed that, as MDD is a complex biological system disorder and that a reductionist approach has not led to any definitive screening or diagnostic biomarkers for MDD, integrals and differentials of profiles of biomarkers can generate appropriate screening biomarkers for MDD.

MDD causes disruption to the Psycho-immuno-neuro-endocrine (PINE) network with dysfunction in immunc, autonomic, metabolic and endocrine functionality (Stapelberg et al., 2015). Therefore, MDD may be characterized by a disruption of multiple biomarkers, which reflect multiple changes in the complex pathophysiological system underlying MDD. The effect of MDD on various biological systems has been the focus of research for several decades but the search for a single objective test has been fruitless (Kunugi et al., 2015) so it is proposed that a profile of biomarkers reflecting changes in absolute levels of biomarkers, in responsiveness and reactivity of systems, and/or changes in regulatory set points around which biomarkers vary pathophysiologically (Juster et al., 2010) would have greater clinical utility.

\section{A Mathematical Hierarchy of Predictive Biomarkers}

Scheffer et al (Scheffer et al., 2009) proposed a generic categorical classification for early warning systems for tipping points before they reach catastrophic bifurcations. Changes in biomarkers within the complex systems that are dysfunctional in MDD can be summarized into five categories as categories of changes seen prior to catastrophic bifurcations: 
- skew

- slowing response or resistance to change

- flickering

- increased variance

- autocorrelation.

Additionally, applying Perturbation Theory for developing a methodological framework into biomarker research in MDD, these categories can be simplified into first, second and third derivative differentials and integrals of biomarkers with respect to time. The starting point is the initial absolute level of biomarkers at a single point in time, generating one of the five types of tipping point changes proposed by Scheffer et al, and. can be expressed mathematically as:

The first derivative differential of the rate of change in biomarker concentration is skew (s) where

$$
\mathrm{s}=\mathrm{d}[\mathrm{b}] / \mathrm{dt}
$$

reflects change in the balance of active products or end products within the complex system. For example tryptophan, kynurenine, quinolinic acid and serotonin, where the absolute level or a ratio of absolute levels are the relevant biomarkers. Similarly, skew is reflected in an increase in antithyroperoxidase antibodies (anti-TPO) and anti-thryotropin (anti-TSH) receptor antibodies from baseline over time and are the first derivative differentials.

- The second derivative differential of the rate of change in biomarker concentration is slowing response or resistance to change (r) where

$$
\mathrm{r}=\mathrm{d}^{2}[\mathrm{~b}] / \mathrm{dt}^{2}=\mathrm{ds} / \mathrm{dt}
$$

reflects change in the regulatory sensitivity of feedback loops and control within a complex system, for example, changes to cortisol sensitivity with loss of dexamethasone suppression or elevated early morning/awakening cortisol. 
- The third derivative differential of the rate of change in biomarker concentration is flickering ( $\mathrm{k})$, where

$$
\mathrm{k}=\mathrm{d}^{3}[\mathrm{~b}] / \mathrm{dt}^{3}=\mathrm{dr} / \mathrm{dt}
$$

reflects change in heart rate variability (HRV) and is linked with MDD (Kemp et al., 2010).

- Variance $(\sigma)$ is defined by the area under the curve, or definite integral, of the biomarker concentration $[\mathrm{b}]$ as a function of time, where

$$
\sigma=\int f[b] d t
$$

reflects changes in the operating set points of a complex system and its feedback loops. For example, 24-hour urine metanephrine is the definite integral of catecholamine production over time and $\mathrm{HbAlc}$ is the definite integral of plasma glucose over 3 months with approximately half related to the previous month and the remaining half resulting from elevated glucose levels in the $2^{\text {nd }}$ and $3^{\text {rd }}$ months of the red cell's life (Burtis et al., 2015b).

- The Laplace transformation of the biomarker concentration is autocorrelation (a) and is seen as repeating patterns of flickering or change in variance. It can be positive or negative where there is increasing or reducing amplitude of episodes of flickering or variance respectively. For screening purposes, positive autocorrelation indicates that resistance to change has occurred and the biological system under analysis has changed from a negative feedback inhibition controlled system to one showing impaired control and markedly changed response to stimuli. It reflects moving-average model from changes in sensitivity of feedback loops resulting in repeating patterns of altered responses 
to changes over time and here $\rho$ is the frequency of changes in biomarker concentration $[\mathrm{b}]$ :

$$
a=\int f(t) e^{-\rho t} d t
$$

For example, cortisol shows episodic release, dynamic responsiveness to environmental and internal stimuli as well as a diurnal rhythm. The Trier Social Stress Test (TSST) shows responses to stress and recovery from it. Repeated TSSTs would show autocorrelation, system responsiveness and dysfunctional system reactivity and recovery (Kirschbaum et al., 2008, Campbell ând Ehlert, 2012). Similarly, repeated $\mathrm{HbA} 1 \mathrm{c}$ estimations would reveal autocorrelation or recurring patterns of increased variance and change in the moving average. Again, $5 \mathrm{HT}_{1 \mathrm{~A}}$ receptors are up- and down-regulated in response to rates of change in serotonin activity (Zhang et al., 2014, Andrews et al., 2015). So, measuring receptor activity over time reveals autocorrelation as the end response to acceleration and deceleration of the rate of change of serotonin activity on cells.

\section{Results}

\section{Proposed Biomarkers in MDD}

Biomarkers were identified from reviews of MDD from 2010 where there was evidence of dysfunction in depression (Papakostas et al., 2011, Boksa, 2013, Ebot Enaw and Smith, 2013, Lang and Borgwardt, 2013, Lopresti et al., 2014, Seib et al., 2014, Bilello et al., 2015, Kunugi et al., 2015, Young et al., 2016, Zheng et al., 2016, Harada et al., 2017, Machado-Vieira et al., 2017, Strawbridge et al., 2017). The mathematical models were applied to the biomarkers identified and, if a biomarker had the potential to reflect one of the five patterns seen before a catastrophic bifurcation, it was included in 
the model as a proposed tipping point biomarker. All other biomarkers were excluded, specifically, those that did not have the potential to reflect one of the five patterns occurring before catastrophic bifurcations or those that did not reflect changes in the two extracellular and two intracellular systems identified. Allocation of biomarkers to the intracellular and extracellular subgroups was arbitrary because many of the biomarkers are involved in feedback loops in more than one subgroup. For example, tumour necrosis factor alpha (TNF $\alpha)$ is involved in activation of the HPA axis, stimulates indole dioxygenase in tryptophan metabolism, and activates proinflammatory cytokines as well as the serotonin (5-HT) transporter. Biomarkers were grouped extracellularly or intracellularly and were further subdivided into the two most relevant domains that are dysfunctional in MDD:

- Extracellularly, HPA axis dysfunction and metabolic dysfunction

- Intracellularly, altered tryptophan/kynurenine/serotonin metabolism, and inflammation and mitochondrial oxidative stress.

The list of proposed biomarkers is not exhaustive and is given in Tables 1 and 2 .

Evidence supporting biomarker utility in screening for MDD

Extracellular Tipping Point Biomarkers

A. HPA Dysfunction and Glucocorticoid Resistance

\section{Salivary Cortisol}

Dysfunctional activity of the HPA axis with chronic stress is derived from its harmful effects on dendritic processes, impaired neurogenesis, reduced neuroplasticity and neuronal loss (Sapolsky, 2000). There is no standardised guidance on measurement and use of salivary cortisol (Ryan et al., 2016) but there has been a user's guide developed to assist researchers and practitioners (Hayes et al., 2016), which may help reduce 
variability of results found in studies. The area under the curve and integral of early morning salivary cortisol have been recommended to improve sensitivity and specificity of the biomarkers (Lu et al., 2016, Zhang et al., 2016). In an analysis of results of a 34study meta-analysis and stress, difficulty comparing results and interpreting the data led to the proposal that the integral of salivary cortisol is used to reduce methodological variation and variability of results (Liu et al., 2017).

Reduced feedback inhibition and glucocorticoid resistance are seen with early childhood trauma and the integral of the cortisol awakening response was used to show hyper-reactivity of the HPA axis in MDD (Lu et al., 2016). In contrast, the integral of the early morning awakening response over 90 minutes in young males was reduced when family conflict when measured by the family environment scale (Zhang et al., 2016). Early morning salivary cortisol was found to be a valid screening biomarker for MDD (Owens et al., 2014) and the biomarker appeared to differentiate depressive symptoms from anxiety (Nelemans et al., 2014). The initial physiological response to stress, as seen in increased salivary cortisol, reduces over time (Miller et al., 2007) and is affected by genotype where people with the 5HTTLPR L allele have a greater response than those with the SS allele (Ancelin et al., 2017). Salivary cortisol is unaffected by age, weight, smoking, sleep duration, time of awakening or alcohol consumption (Pruessner et al., 1997, Dmitrieva et al., 2013).

Glucocorticoid Receptor (Nuclear Receptor Subfamily 3 Group C Member) Nuclear receptor subfamily three group C member 1 (NR3C1) or Glucocorticoid Receptors (GR) are implicated in the aetiology of depression (Holsboer, 2000, Sapolsky, 2000) and are noted to be hyper-methylated in type II diabetes (Nontharat, 2015, Ouakinin, 2016). Hyper-methylation appears to be an epigenetic change which is a mediator of early childhood adversity related disorders (Tsankova et al., 2007, 
Covington et al., 2009). Glucocorticoid resistance is maintained in part by proinflammatory biomarkers that reduce glucocorticoid receptor functionality (Pariante and Lightman, 2008b). Feedback responsiveness in the HPA system is improved from glucocorticoid resistance to normal feedback inhibition with antidepressant therapy (Pariante and Lightman, 2008b).

\section{Combined Dexamethasone Suppression Test/Corticoliberin Stimulation Test}

HPA axis dysregulation occurs in depressed patients exhibiting elevated cortisol, corticoliberin $(\mathrm{CRH})$, impaired suppression of the dexamethasone suppression test, and a blunted adrenocorticotrophic hormone (ACTH) response to CRH (Aihara et al., 2007, Schüle et al., 2009). Evidence of HPA axis activation appears to have prognostic value and is associated with increased risk of depression relapse and even suicide (Varghese and Brown, 2001). In addition, hypercortisolism is linked to both insulin resistance and major depression (Weber et al., 2000). Cortisol, the combined CRH/Dexamethasone Suppression Test (DST) test and the HPA axis have been reviewed by Young et al indicating potential as biomarkers in depression and to help reveal tipping transformations in HPA axis dysfunction (Young et al., 2016).

Trier Social Stress Test (TSST) and $\mathrm{CO}_{2}$ Stress Test

The TSST and $\mathrm{CO}_{2}$ stress tests both activate cortisol and the human stress response with pre-response sensitivity to cortisol and post-stress resistance to cortisol (Kirschbaum et al., 2008, Lu et al.,2016). Early trauma also leads to increased activation of adult HPA axis and immune systems demonstrated with increased biomarkers TNF $\alpha$, interleukin- 6 (IL-6), IL-1 $\beta$, and C-reactive protein (CRP) (Liu et al., 2017). 


\section{B. Metabolic Dysfunction}

Acute Phase Response

CRP has been shown to be elevated in depressed patients (Dowlati et al., 2010). In the acute phase response Cortisol Binding Globulin (CBG) falls while CRP rises (Burtis et al., 2015a). The ratio would increase sensitivity and demonstrate early development of an acute phase response making it one of the potential screening biomarker in depression.

Hypothalamo-Pituitary-Thyroid Axis (HPT Axis) and Autoimmunity Thyroid stimulating hormone (TSH) fluctuates in a nyctohemeral rhythm (Sviridonova et al., 2012) with a peak at 0200-0400 hours and trough at 1600-2000 hours (Persani et al., 1995).

An increase in antithyroperoxidase antibodies (anti-TPO Abs) has been associated with MDD suggesting its use as a biomarker (Van de Ven et $a l, 2012$ ). A higher lifetime prevalence of depression was found in anti-TPO antibody positive versus negative individuals (Dufour, 2007). There is a reported association between anti-TPO Abs and lifetime diagnosis for major depressiye disorder (Carta et al., 2004b). Anti-TPO Abs are also associated with increased risk of hypothyroidism, a known causative factor in MDD (Marangell and Callahan, 1998, Carta et al., 2004a, Fountoulakis et al., 2004).

Low TSH in normal individuals appears to be linked to an increased rate of depression (Dayan and Panicker, 2009, Estrada et al., 2014). Intracellular type 1 deiodinase determines thyroxine:tri-iodothyronine (T4:T3) ratio and phosphodiesterase determines TSH levels while both of these are connected to psychological well-being (Dayan and Panicker, 2009). 
Hair T3, the long-term integral of plasma T3, the active hormone, has been shown to be significantly lower in depressed patients (Paus et al., 2014, Wei et al., 2014).

\section{Leptin and Ghrelin}

Ghrelin causes increased intracellular levels of reactive oxygen species (ROS) (Buldak RJ et al., 2015) and is associated with oxidative stress and insulin resistance (Razzaghy-Azar et al., 2016). In addition, women with interpersonal stress had higher ghrelin and lower leptin levels, suggesting the ratio would be more revealing than either biomarker concentration alone (Jaremka et al., 2014). Leptin levels have also been shown to be significantly associated with low mood in normal weight women (Häfner et al., 2012). Leptin and glucocorticoid receptors are co-located on hippocampal progenitor cells and act on neurogenesis through Glycogen synthase kinase (GSK-3)

(Garza et al., 2012).

Heart rate variability

Heart rate variability (HRV) is an established measure of cardiac vagal control (Rottenberg, 2007). Flickering reflects increasing asymmetry of fluctuations over time before a critical transition, which can theoretically be measured by assessing HRV. Flickering could be investigated using the R-wave interval time series in a 24 hour cardiac recording to predict underlying change in autonomic function. The low frequency component of RR-interval data has been used to analyse the stochastic change with persistence around a set-point, which relates to sympathetic and parasympathetic cardiac control (Zheng et al. (2013), and it is proposed that similar methodology could be used to detect a critical transition to MDD. 
Carbohydrate metabolism and Haemoglobin $A_{l c}\left(H b A_{l c}\right)$

Insulin resistance or impaired glucose tolerance, as indicated by the oral glucose tolerance test, fasting plasma glucose and $\mathrm{HbA}_{\mathrm{tc}}$, have been linked to depression (Winokur et al., 1988, Weber et al., 2000, Chen et al., 2010). $\mathrm{HbA}_{1 \mathrm{c}}$ is the area under the curve or integral of the average plasma glucose over the preceding 8-12 weeks, if red cell survival is normal. Approximately $50 \%$ of $\mathrm{HbA}_{1 \mathrm{c}}$ is derived from the plasma glucose over the preceding month and the remainder from the two months before that (Burtis et al., 2015a). Increased variance is seen when the range of concentrations of glycated haemoglobin measured over time increases above baseline. It indicates that the plasma glucose concentration is, at times, elevated above the normal physiologically controlled range. These raised levels may not be detected by measuring random plasma glucose levels as they can be transitory. However, irreversible, non-enzymatic binding of glucose to the N-terminal valine amino acid on the beta globin chains on haemoglobin A occurs directly in proportion to the plasma glucose concentration. As it forms a stable ketoamine over the life of the red cell, the concentration of glycated $\mathrm{HbA}_{1 \mathrm{c}}$ detects those elevations, the increased variance in the plasma glucose and the area under the curve of plasma glucose concentrations over the life of that red cell. Human leukocyte telomere length (LTL)

There is an association between major depressive disorder and telomere length ( $p$ $<0.001)$ and with severity of depression $(\mathrm{p}=0.03)$ (Ridout et al., 2016). Concurrent telomere length was more strongly associated than longitudinal telomere length suggesting more recent epigenetic change. The current hypothesis is that oxidative stress exposure and ageing lead to reduction in telomere length. 


\section{Intracellular Tipping Point Biomarkers}

A Dysfunction of tryptophan metabolism in the serotonin/kynurenine/quinolinic acid pathways

\section{Tryptophan Metabolism}

Tryptophan is an essential amino acid and therefore supply is rate limiting if intake is inadequate. Tryptophan can only be transported across the blood brain barrier in its free form by a competitive and non-specific amino acid transporter (Chen and Guillemin, 2009). It then is incorporated into proteins or converted into serotonin or kynurenine. $99 \%$ of dietary tryptophan is metabolised down the kynurenine pathway (Mbongue et $a l ., 2015)$. Indolamine 2, 3-dioxygenase (IDU) is the tirst and rate-limiting enzyme of tryptophan metabolism through the kynurenine pathway. Alternatively, tryptophan can be metabolised in neurones to serotonin with the initial rate limiting enzyme tryptophan hydroxylase. Serotonin is metabolised to 5HIAA while kynurenine is metabolised to kynurenic acid, picolinic acid or quinolinic acid. In turn, quinolinic acid is converted to Nicotinamide Adenine Dinucleotide (NAD) and NAD phosphate (NADP) that is required for oxidative metabolism directly linking tryptophan supply to mitochondrial oxidative phosphorylation and intracellular energy production (Ruddick et al., 2006). In states of increased consumption of tryptophan such as inflammation, production of serotonin is limited by necessity for protein synthesis and production of NAD and NADP, and this pathway is metabolically prioritised (Lundstrom et al., 2005, Ruddick et al., 2006). The ratio of kynurenine: tryptophan is an indicator for the activity of IDO (Mbongue et al., 2015), which is an immunomodulatory enzyme (Ruddick et al., 2006). Measurement of the differentials and integrals of kynurenine metabolites, including those as ratios to serotonin metabolites, can serve as biomarkers for activity of these related, but competing, pathways. 


\section{Leucocyte $5 H T_{1 A}$ receptors}

In depressed patients, $5-\mathrm{HT}_{1 \mathrm{~A}}$ receptor expression on leucocytes is inversely correlated with serotonin activity in plasma and 5-hydroxyindoleacetic acid (5-HIAA) concentration in urine (the end-product of serotonin metabolism). This occurs through a negative feedback mechanism and affects the HPA axis (Zhang et al., 2014).

Resistance to change is the second derivative differential, where the rate of change in concentration of a biomarker is measured. Practically, that would require multiple measurements, which can be clinically untenable. However, measuring the white cell response to the alterations in the rate of change, in this case resistance to negative feedback inhibition and down-regulation of receptor expression, is a biomarker reflecting the effect of changes in the velocity of $5 \mathrm{HT}_{1 \mathrm{~A}}$ concentration pathophysiologically.

\section{Galanin}

Galanin is found in gut and brain tissue. It is an inhibitory neurotransmitter co-located with serotonin and noradrenaline, with the highest concentrations in the CNS found in the hippocampus where it acts as an inhibitory neuromodulator modifying glutamate, but not GABA transmission. It has been linked to depression and anxiety through modulation of monoamine transmitters and the neuroendocrine system (Kozlovsky et al., 2009) (Lundstrom et al., 2005). Galanin also stimulates the HPA axis increasing CRH and ACTH release and modulating the HPA axis (Tortorella et al., 2007). In addition, there are Galanin receptors in the adrenal medulla, so it is involved in regulation of the autonomic nervous system (Tortorella et al., 2007).

\section{B Inflammation and Mitochondrial Oxidative Stress}

Oxidative stress has been implicated in the pathophysiology of MDD and can be reversed by serotonin reuptake inhibitors (Bilici et al., 2001, Khanzode et al., 2003). 
The free radical theory proposes that ROS produce mitochondrial damage, which in turn reduces energy supply through impaired oxidative phosphorylation. Damaged proteins then activate mechanistic target of rapamycin (mTOR) for autophagy, further potentiating long term cellular senescence (Kriete et al., 2010).

Tumour Necrosis Factor (TNF $\alpha)$

TNF $\alpha$ has been proposed as a biomarker in MDD reflecting HPA axis dysfunction (Tanabe and Nomura, 2007, Berthold-Loslben and Himmmerich, 2008). It has also been associated with activation of the cytokine system and the behavioural changes associated with major depressive disorder and sickness behaviour, including malaise, pyrexia, social isolation, anhedonia, anorexia, loss of concentration and lethargy (Reichenberg et al., 2001). Cytokine activation leads to activation of serotonin transporters and relative serotonin deficiency (Zhu et al., 2006). This is mediated by TNF $\alpha$ and stimulation of IDO with resultant depletion of tryptophan (Wichers and Maes, 2002). TNF $\alpha$ blockers used in the treatment of psoriasis are associated with more than $50 \%$ improvement in Hamilton Depression Scale, Beck Depression Inventory and Chronic Illness Therapy Fatigue Scale, and with reduction in sickness behaviour (Wichers and Maes, 2002). Obesity increases TNF $\alpha$ over time leading to sickness behaviours similar to those seen in MDD (Ouakinin, 2016).

$N F-\kappa B$

Nuclear factor kappa-light-chain-enhancer of activated B cells (NF- $\mathrm{KB})$ is a protein complex that controls transcription of deoxyribonucleic acid (DNA), cytokine

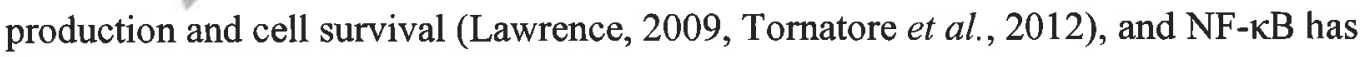
also been implicated in synaptic plasticity and memory (Albensi and Mattson, 2000, Meffert et al., 2003). NF-kB is a first responder to harmful cellular stimuli (Vlahopoulos et al., 2015). Known inducers of NF- $\mathrm{\kappa B}$ activity are highly variable and 
include ROS and TNF $\alpha$ (Chandel et al., 2000). Fidelity of feedback responses between diverse cell types and the immune system depends on the integrity of mechanisms that limit the range of genes activated by NF- $\mathrm{KB}$, allowing sole expression of genes which contribute to an effective immune response and subsequently, a complete restoration of tissue function after resolution of inflammation (Vidal et al., 2012). It is understood that chronic low-grade inflammation plays a key role in the initiation, propagation, and development of metabolic diseases consistent with its central role in coordinating inflammatory responses, numerous recent studies have implicated the transcription factor NF-kB in the development of such diseases (Baker et al., 2011). Several studies during the past two decades have highlighted the key role of the inhibitor of kappa B kinase (IKK)/NF-kB pathway (Israël, 2010) in the induction and maintenance of the state of inflammation that underlies metabolic diseases such as obesity and type 2 diabetes (Hayden and Ghosh, 2008).

Zinc and Magnesium

Both $\mathrm{Zn}$ and $\mathrm{Mg}$ are known to inhibit the NMDA glutamate receptor complex and improve mood in depression (Barragan-Rodriguez et al., 2008, Sawada and Yokoi, 2010) and could form part of a profile of biomarkers in screening for MDD.

Brain Derived Neurotrophic Factor (BDNF)

BDNF is critically involved in neuroplasticity in the neural networks giving rise to sickness behaviours and behaviours in depression (Schinder and Poo, 2000, Pittenger and Duman, 2008). BDNF signals through glycogen synthase kinase 3 (GSK-3) (Ricken et al., 2013). BDNF levels are noted to be reduced in the serum of depressed people, and increase in response to antidepressant therapy (Sen et al., 2008, Molendijk et al., 2014). Higher BDNF levels were also associated with better treatment outcomes. 
Glycogen Synthase Kinase 3 (GSK-3)

GSK-3 is protein kinase and active in a number of central intracellular signalling pathways, including cellular proliferation, migration, glucose regulation, and apoptosis. Due to its importance across numerous cellular functions, GSK-3 activity is subject to tight regulation (Cole and Sutherland, 2008). The activity of GSK-3 is far greater in the nucleus and mitochondria than in the cytosol in cortical neurons (Bijur and Jope, 2003). Lithium is an inhibitor of GSK-3 and is an effective mood stabiliser in Bipolar Disorder, and as an augmenting agent in treatment refractory MDD (Taylor et al., 2015). Other GSK-3 inhibitors show promise in the treatment of type 2 Diabetes Mellitus, which is, in itself, correlated with depression (Rayasam et al., 2009). Cole and Sutherland have reviewed available methods and concluded that the challenge with GSK-3 measurements is that different methods give significantly disparate results (Cole and Sutherland, 2008).

Circular RNAs

Circular, covalently linked segments of RNA in plasma have been identified as having utility as novel biomarkers in neurological disorders and depression (Cui et al., 2016, Ma et al., 2016).

Mechanistic Target of Rapamycin (mTOR)

MTOR is a protein kinase that regulates cell growth, energy production, cell survival and autophagy. It is a central intracellular regulator of energy production, oxygen levels and nutrient use (Tokunaga et al., 2004). It is implicated in MDD through control of cellular metabolism (Tokunaga et al., 2004), and ketamine, an N-Methyl-D-Aspartic acid (NDMA) antagonist and its metabolite Nor-Hydroxy-Ketamine (NHK), an Aminohydroxy-Methyl-isoxazole-Propanoic Acid (AMPA) agonist, (Zanos et al., 2016, Machado-Vieira et al., 2017), may act as antidepressants through rapid activation of the 
mTOR pathway, increasing spine formation and synaptic transmission in the prefrontal cortex (Welberg, 2010) (Li et al., 2010). Dysfunctional activity of mTOR has been associated with reduced synaptic plasticity and impaired learning and memory (Oddo, 2012), one of the six cognitive domains impaired in MDD revealed as difficulty with decision making and learning new tasks (American Psychiatric Association, 2013). Reduced levels of mTOR are associated with increased cell lifespan, improved glycolysis and decreased removal of dysfunctional intracellular components (Lang and Borgwardt, 2013).

Interleukin $6(I L-6)$ and $I L-10$

Repeated IL6:IL10 may show a change to a critical transition by revealing a change to a pro-inflammatory state to through positive autocorrelation. IL-10 is one of the antiinflammatory family of cytokines (Oral et al., 2006) in contrast to IL-6 which is proinflammatory cytokine (Heinrich et al., 2003) and anti-inflammatory myokine (Pedersen and Febbraio, 2008) and these interleukins have a role in modulation of neuronal plasticity (Vidal et al., 2012).

Sickness behaviour has been shown to be mediated by pro-inflammatory cytokines (Dantzer, 2009). IL-6 and TNF $\alpha$ have been shown to be elevated in a metaanalysis of studies on depressed patients (Dowlati et al., 2010). Cytokines have been also found to be related to low mood in males (Chen et al., 2010).

Measuring IL6 and IL10 levels multiple times can show a change from a balanced inflammatory state and normal predominance of anti-inflammatory activity, to a pro-inflammatory state with pro-inflammatory biomarkers elevated. A ratio can detect this change earlier improving sensitivity, which is an advantage for a screening biomarker. 
Salivary cortisol and IL-6 were linked to MDD in an 8 study meta-analysis of patients on interferon alpha (IFN- $\alpha$ ) therapy (Machado et al., 2017)

\section{Discussion}

The four functions of biomarkers (screening, diagnosis, prognostic and monitoring) have inherently differing characteristics. By focussing on those for screening, and applying CBP theories and Scheffer et al's five generic categories of tipping point transformations that occur before catastrophic bifurcations, four groups of biomarkers, and the integrals and differentials of those, were identified. They were identified and divided into extracellular and intracellular tipping point biomarkers:

Extracellularly - HPA axis and metabolic dysfunction

Intracellularly - dysfunction of tryptophan metabolism, and inflammation and mitochondrial oxidative stress.

Potential screening biomarkers were proposed, serving as surrogates for the tipping points as listed in Tables $1 \&$ 2.It is the combination of a group of biomarkers that has the potential to lead to a screening profile to aid clinicians in screening for MDD in high risk patients.

The approach used here can also be applied to diagnostic, prognostic and monitoring biomarkers but will, in all probability, result in a differing profile of biomarkers for each function.

\section{Conclusion}

Further biomarker research is reliant on research into the physiology and pathology of MDD. It is proposed that the next steps in the research of biomarkers in MDD are to develop and test a comprehensive list of target biomarkers for the tipping points in 
MDD by identifying relevant system specific biomarkers that fulfil the essential

characteristics for screening, as opposed to diagnosis, prognosis or monitoring response

to therapy. The use of the theoretical approach and mathematical framework to identify

biomarker changes in the tipping points prior to catastrophic bifurcations will

potentially aid in screening, diagnosis, prognosis and monitoring of MDD.

\section{Declaration of Interests}

The authors report no conflicts of interest.

This research did not receive any specific grant from funding agencies in the public, commercial, or not-for-profit sectors.

\section{References}

Aihara, M., Ida, I., Yuuki, N., Oshima, A., Kumano, H., Takahashi, K., Fukuda, M., Oriuchi, N., Endo, K., Matsuda, H. \& Mikuni, M., 2007. HPA axis dysfunction in unmedicated major depressive disorder and its normalization by pharmacotherapy correlates with alteration of neural activity in prefrontal cortex and limbic/paralimbic regions. Psychiatry Research: Neuroimaging, 155, 245256.

Albensi, B.C. \& Mattson, M.P., 2000. Evidence for the involvement of TNF and NFkappaB in hippocampal synaptic plasticity. Synapse, 35, 151-9.

American Psychiatric Association, 2013. Diagnostic and statistical manual of mental disorders Washington: American Psychiatric Association.

Ancelin, M., Scali, J., Norton, J., Ritchie, K., Dupuy, A., Chaudieu, I. \& Ryan, J., 2017. The effect of an adverse psychological environment on salivary cortisol levels in the elderly differs by 5-HTTLPR genotype. Neurobiol Stress, 7, 38-46.

Andrews, P.W., Bharwani, A., Lee, K.R., Fox, M. \& Thomson, J.A., Jr., 2015. Is serotonin an upper or a downer? The evolution of the serotonergic system and its role in depression and the antidepressant response. Neurosci Biobehav Rev, 51, 164-88.

Arnold, V.\& Novikov, S., 1994. Dynamical Systems V, Encyclopedia of Mathematical Sciences, Vol. 5. Springer-Verlag.

Baker, R.G., Hayden, M.S. \& Ghosh, S., 2011. NF-kB, Inflammation, and Metabolic Disease. Cell Metabolism, 13, 11-22.

Barragan-Rodriguez, L., Rodriguez-Moran, M. \& Guerrero-Romero, F., 2008. Efficacy and safety of oral magnesium supplementation in the treatment of depression in the elderly with type 2 diabetes: a randomized, equivalent trial. Magnes Res, 21, 218-23.

Berthold-Loslben, M. \& Himmmerich, H., 2008. The TNF Alpha System: functional aspects in depression narcolepsy and psychopharmacology. Current Neuropharmacology, 6, 193-202. 
Bijur, G. \& Jope, R., 2003. Glycogen synthase kinase-3 beta is highly activated in nuclei and mitochondria. Neuroreport, 14, 2415-9.

Bilello, J., Thurmond, L., Smith, K., Pi, B., Rubin, R., Wright, S., Taub, F. \& Al., E., 2015. MDDScore. The Journal of Clinical Psychiatry, e199-e206.

Bilici, M., Efe, H., Koroglu, M., Uydu, H., Bekaroglu, M. \& Deger, O., 2001. Antioxidative enzyme activities and lipid peroxidation in major depression: alterations by antidepressant treatments. J Affect Disord, 64, 43-51.

Boeing, G., 2016. Visual Analysis of Nonlinear Dynamical Systems: Chaos, Fractals, Self-Similarity and the Limits of Prediction. Systems, 4, 37.

Bogolyubov, N.N.J.O., 2011. Perturbation Theory. The Encyclopedia of Mathematics.

Boksa, P., 2013. A way forward for research on biomarkers for psychiatric disorders. Journal of Psychiatry \& Neuroscience, 38, 75-55.

Buldak Rj, Pilc-Gumula K, Buldak L, Witkowska D, Kukla M, R, P. \& K., Z.-K., 2015. Effects of ghrelin, leptin and melatonin on the levels of reactive oxygen species, antioxidant enzyme activity and viability of the HCT 116 human colorectal carcinoma cell line. Mol Med Rep, 12, 2275-82.

Burtis, C., Bruns, D., Sawyer, B. \& Tietz, N., 2015a. Tietz fundamentals of clinical chemistry and molecular diagnostics.

Burtis, C.A., Bruns, D.E., Sawyer, B.G. \& Tietz, N.W., 2015b. Tietz fundamentals of clinical chemistry and molecular diagnostics.

Campbell, J. \& Ehlert, U., 2012. Acute psychosocial stress: does the emotional stress response correspond with physiological responses? Psychoneturoendocrinology, 37, 1111-34.

Carta, M.G., Loviselli, A., Hardoy, M.C., Massa, S., Cadeddu, M., Sardu, C., Carpiniello, B., Dell'osso, L. \& Mariotti, S., 2004a. The link between thyroid autoimmunity (antithyroid peroxidase autoantibodies) with anxiety and mood disorders in the community: a field of interest for public health in the future. BMC Psychiatry, 4, 25.

Carta, M.G., Loviselli, A., Hardoy, M.C., Massa, S., Cadeddu, M., Sardu, C., Carpiniello, B., Dell'osso, L. \& Mariotti, S., 2004b. The link between thyroid autoimmunity (antithyroid peroxidase autoantibodies) with anxiety and mood disorders in the community: a field of interest for public health in the future. BMC Psychiatry, 4, 25.

Chandel, N.S., Trzyna, W.C., Mcclintock, D.S. \& Schumacker, P.T., 2000. Role of oxidants in NF-kappa B activation and TNF-alpha gene transcription induced by hypoxia and endotoxin. J Immunol, 165, 1013-21.

Chen, Y. \& Guillemin, G., 2009. Kynurenine Pathway Metabolites in Humans: Disease and Healthy States. International Journal of Tryptophan Research : IJTR, 2, 119.

Chen, Y., Lin, W., Chen, Y., Mao, W. \& Hung, Y., 2010. Antidepressant Effects on Insulin Sensitivity and Proinflammatory Cytokines in the Depressed Males. Mediators of Inflammation, 2010, 7.

Cole, A. \& Sutherland, C., 2008. Measuring GSK3 expression and activity in cells. Methods Mol Biol, 468, 45-65.

Covington, H., Maze, I., Laplant, Q., Vialou, V., Ohnishi, Y., Berton, O., Fass, D., Renthal, W., Rush, A., Wu, E., Ghose, S., Krishnan, V., Russo, S., Tamminga, C., Haggarty, S. \& Nestler, E., 2009. Antidepressant actions of histone deacetylase inhibitors. $J$ Neurosci, 29, 11451-60.

Cui, X., Niu, W., Kong, L., He, M., Jiang, K., Chen, S., Zhong, A., Li, W., Lu, J. \& Zhang, L., 2016. hsa_circRNA_103636: potential novel diagnostic and 
therapeutic biomarker in Major depressive disorder. Biomarkers in Medicine, 10, 943-952.

Dantzer, R., 2009. Cytokine, sickness behavior, and depression. Immunol Allergy Clin North Am, 29, 247-64.

Dayan, C. \& Panicker, V., 2009. Novel insights into thyroid hormones from the study of common genetic variation. Nat Rev Endocrinol, 5, 211-8.

De Long, C., 2016. The elusive blood test for depression. . Clincal Laboratory News. https://www.aacc.org/publications/cln/articles/2016/march/the-elusive-bloodtest-for-depression.: AACC.

Dmitrieva, N., Almeida, D., Dmitrieva, J., Loken, E. \& Pieper, C., 2013. A daycentered approach to modeling cortisol: diurnal cortisol profiles and their association among U.S. adults. Psychoneuroendocrinology, 2354-65.

Dowlati, Y., Herrmann, N., Swardfager, W., Liu, H., Sham, L., Reim, E. \& Lanctot, K., 2010. A meta-analysis of cytokines in major depression. Biol Psychiatry, 67. 446-57.

Dufour, D.R., 2007. Laboratory Tests of Thyroid Function: Uses and Limitations. Endocrinology and Metabolism Clinics of North America, 36, 579-594.

Ebot Enaw, J. \& Smith, A., 2013. Biomarker development for brain-based disorders: recent progress in psychiatry. $J$ Neurol Psychol, 1, 7.

Estrada, J., Soldin, D., Buckey, T., Burman, K. \& Soldin, O., 2014. Thyrotropin Isoforms: Implications for Thyrotropin Analysis and Clinical Practice. Thyroid, $24,411-423$

Ferrari, A., Somerville, A., Baxter, A., Norman, R., Patten, S., Vos, T. \& Whiteford, H., 2012. Global variation in the prevalence and incidence of major depressive disorder: a systematic review of the epidemiological literature. Psychological Medicine, 43, 471-481.

Fountoulakis, K.N., Iacovides, A. \& Grammaticos, P., 2004. Thyroid function in clinical subtypes of major depression.

Garza, J., Guo, M., Zhang, W. \& Lu, X., 2012. Leptin restores adult hippocampal neurogenesis in a chronic unpredictable stress model of depression and reverses glucocorticoid-induced inhibition of GSK-3beta/beta-catenin signaling. $\mathrm{Mol}$ Psychiatry, 17, 790-808.

Häfner, S., Baumert, J., Emeny, R., Lacruz, M., Thorand, B., Herder, C., Koenig, W. \& $A l, E$. 2012. Sleep disturbances and depressed mood: A harmful combination associated with increased leptin levels in women with normal weight. Biological Psychology, 89, 163-169.

Harada, P.H., Buring, J.E., Cook, N.R., Cobble, M.E., Kulkami, K.R. \& Mora, S., 2017. Impact of Subclinical Hypothyroidism on Cardio Metabolic Biomarkers in Women. Journal of the endocrine Society, 1 (2): 113-1 to 3.

Hayden, M.S. \& Ghosh, S., 2008. Shared principles in NF-kappaB signaling. Cell, 132, $344-62$.

Hayes, L., Sculthorpe, N., Cunniffe, B. \& Grace, F., 2016. Salivary testosterone and cortisol measurement in sports medicine: a narrative review and user's guide for researchers and practitioners. Int J Sports Med, 37, 1007-1018.

Heinrich, P., Behrmann, I., Haan, S., Hermanns, H., Müller-Newen, G. \& Schaper, F., 2003. Principles of interleukin (IL)-6-type cytokine signalling and its regulation. Biochemical Journal, 374, 1.

Holsboer, F., 2000. The corticosteroid receptor hypothesis of depression. Neuropsychopharmacology, 23, 477-501. 
Israël, A., 2010. The IKK Complex, a Central Regulator of NF-kB Activation. Cold Spring Harbor Perspectives in Biology, 2, a000158.

Jaremka, L., Belury, M., Andridge, R., W, M., Glaser, R., Christian, L., Emery, C. \& $A l ., E ., 2014$. Interpersonal stressors predict ghrelin and leptin levels in women. Psychoneuroendocrinology, 48, 178-88.

Juster, R.-P., Mcewen, B. \& Lupien, S., 2010. Allostatic load biomarkers of chronic stress and impact on health and cognition. Neuroscience \& Biobehavioral Reviews, 35, 2-16.

Kemp, A., Quintana, D., Gray, M., Felmingham, K., Brown, K. \& Gatt, J., 2010. Impact of depression and antidepressant trealment on hedrt rate variability: a review and meta-analysis. Biological Psychiatry, 67, 1067-1074.

Kessler, R., 2012. The costs of depression. Psychiatric Clinics of North America, 35, 114.

Kessler, R., Berglund, P., Demler, O., Jin, R., Koretz, D., Merikangas, K., Rush, A. \& Al., E., 2003. The epidemiology of major depressive disorder. JAMA, 289, 3095.

Khanzode, S.D., Dakhale, G.N., Khanzode, S.S., Saoji, A. \& Palasodkar, R 2003. Oxidative damage and major depression: the potential antioxidant action of selective serotonin re-uptake inhibitors. Redox Rep, 8, 365-70.

Kirschbaum, C., Pirke, K.-M. \& Hellhammer, D., 2008. The 'Trier Social Stress Test' a tool for investigating psychobiological stress responses in a laboratory setting. Neuropsychobiology, 28, 76-81.

Kozlovsky, N., Matar, M., Kaplan, Z., Zohar, J. \& Cohen, H., 2009. The role of the galaninergic system in modulating stress-related responses in an animal model of posttraumatic stress disorder. Biol Psychiatry, 65, 383-91.

Kriete, A., Bosl, W.J. \& Booker, G., 2010. Rule-Based Cell Systems Model of Aging using Feedback Loop Motifs Mediated by Stress Responses. PLOS Computational Biology, 6, e1000820.

Kunugi, H., Hori, H. \& Ogawa, S., 2015. Biochemical markers subtyping major depressive disorder. Psychiatry and Clinical Neurosciences, 69, 597-608.

Lang, U. \& Borgwardt, S., 2013. Molecular mechanisms of depression: perspectives on new treatment strategies. Cell Physiol Biochem, 31, 761-77.

Lawrence, T., 2009. The Nuclear Factor NF-kB Pathway in Inflammation. Cold Spring Harbor Perspectives in Biology, 1, a001651.

Li, N., Lee, B., Liu, R., Banasr, M., Dwyer, J., Iwata, M., Li, X. \& Al, E., 2010. mTORdependent synapse formation underlies the rapid antidepressant effects of NMDA antagonists. Science, 329, 959-64.

Liu, J.J., Ein, N., Peck, K., Huang, V., Pruessner, J. \& Vickers, K., 2017. Sex differences in salivary cortisol reactivity to the Trier Social Stress Test (TSST): a meta-analysis. Psychoneuroendocrinology, 82, 26-37.

Lopresti, A.L., Maker, G.L., Hood, S.D. \& Drummond, P.D., 2014. A review of peripheral biomarkers in major depression: the potential of inflammatory and oxidative stress biomarkers. Prog Neuropsychopharmacol Biol Psychiatry, 48, 102-11.

Lu, S., Gao, W., Huang, M., Li, L. \& Xu, Y., 2016. In search of the HPA axis activity in unipolar depression patients with childhood trauma: Combined cortisol awakening response and dexamethasone suppression test. J Psychiatr Res, 78, 24-30.

Lundstrom, L., Elmquist, A., Bartfai, T. \& Langel, U., 2005. Galanin and its receptors in neurological disorders. Neuromolecular Med, 7, 157-80. 
Ma, K., Guo, L., Xu, A., Cui, S. \& Wang, J.-H., 2016. Molecular Mechanism for StressInduced Depression Assessed by Sequencing miRNA and mRNA in Medial Prefrontal Cortex. PLOS ONE, 11, e0159093.

Machado-Vieira, R., Henter, I.D. \& Zarate, C.A., Jr., 2017. New targets for rapid antidepressant action. Prog Neurobiol, 152, 21-37.

Machado, M., Oriolo, G., Bortolato, B., Kohler, C., Maes, M., Solmi, M., Grande, I. \& A1., E., 2017. Biological mechanisms of depression following treatment with interferon for chronic hepatitis C: A critical systematic review. J Affect Disord, $209,235-245$.

Marangell, B. \& Callahan, A.M., 1998. Mood disorders and the thyroid axis. Current Opinion Psychiatry, 11.

Mayeux, R., 2004. Biomarkers: potential uses and limitations. NeuroRx, 1, 182-8.

Mbongue, C., Nicholas, A., Torrez, W., Kim, N., Firek, F. \& Langridge, H., 2015. The Role of Indoleamine 2, 3-Dioxygenase in Immune Suppression and Autoimmunity. Vaccines, 3.

Meffert, M.K., Chang, J.M., Wiltgen, B.J., Fanselow, M.S. \& Baltimore, D 2003. NFkappa B functions in synaptic signaling and behavior. Nat Neurosci, 6, 1072-8.

Miller, G., Chen, E. \& Zhou, E., 2007. If it goes up, must it come down? Chronic stress and the hypothalamic-pituitary-adrenocortical axis in humans. Psychol Bull, $133,25-45$.

Molendijk, M.L., Spinhoven, P., Polak, M., Bus, B.A., Penninx, B.W. \& Elzinga, B.M., 2014. Serum BDNF concentrations as peripheral manifestations of depression: evidence from a systematic review and meta-analyses on 179 associations (N=9484). Mol Psychiatry, 19, 791-800.

Nelemans, S.A., Hale, W.W., 3rd, Branje, S.J., Van Lier, P.A., Jansen, L.M., Platje, E., Frijns, T., Koot, H.M. \& Meeus, W.H., 2014. Persistent heightened cortisol awakening response and adolescent internalizing symptoms: a 3-year longitudinal community study. J Abnorm Child Psychol, 42, 767-77.

Nontharat, M., 2015. Glucocorticoid receptor gene NR3C1 promoter is hypermethylated In Thai females with Major Depressive Disorder. Genetic Mol Res, 14 (4) 1907-9.

Oddo, S., 2012. The role of mTOR signaling in Alzheimer disease. Frontiers in bioscience (Scholar edition), 4, 941-952.

Oral, H., Kotenko, S., Yılmaz, M., Mani, O., Zumkehr, J., Blaser, K., Akdis, C. \& Al., E., 2006. Regulation of T cells and cytokines by the interleukin-10 (IL-10)family cytokines IL-19, IL-20, IL-22, IL-24 andIL-26. European Journal of Immunology, 36, 380-388.

Ott, E., 2002. Chaos in dynamical systems: Cambridge university press.

Ouakinin, S.R, 2016. Anxiety as a risk factor for cardiovascular diseases. Frontiers in Psychaitry, Issue 7, article 25.

Owens, M., Herbert, J., Jones, P.B., Sahakian, B.J., Wilkinson, P.O., Dunn, V.J., Croudace, T.J. \& Goodyer, I.M., 2014. Elevated morning cortisol is a stratified population-level biomarker for major depression in boys only with high depressive symptoms. Proc Natl Acad Sci U S A, 111, 3638-43.

Papakostas, G., Shelton, R., Kinrys, G., Henry, M., Bakow, B., Lipkin, S., Pi, B. \& Al., E., 2011. Assessment of a multi-assay, serum-based biological diagnostic test for major depressive disorder: a pilot and replication study. Molecular Psychiatry, 18, 332-339.

Pariante, C. \& Lightman, S., 2008a. The HPA axis in major depression: classical theories and new developments. Trends in Neurosciences, 31, 464-468. 
Pariante, M.P. \& Lightman, S.L., 2008b. The HPA Axis in Major Depression: classical theories in new developments will. Trends Neuroscience, 31 (9): 464-8.

Paus, R., Langan, E.A., Vidali, S., Ramot, Y. \& Andersen, B., 2014.

Neuroendocrinology of the hair follicle: principles and clinical perspectives. Trends in Molecular Medicine, 20, 559-570.

Pedersen, B. \& Febbraio, M., 2008. Muscle as an endocrine organ: focus on musclederived interleukin-6. Physiol Rev, 88, 1379-406.

Persani, L., Terzolo, M., Asteria, C., Oriendi, F., Agneli, A. \& Beck Peccoz, P., 1995. Circadian Variation of Fire of Open Bioactivity in Normal Subjects and Patients with Primary Hypothyroidism. Journal of endocrinology and melabolism, 80:2722-28.

Pittenger, C. \& Duman, R., 2008. Stress, depression, and neuroplasticity: a convergence of mechanisms. Neuropsychopharmacology, 33, 88-109.

Pruessner, J., Wolf, O., Hellhammer, D., Buske-Kirschbaum, A., Von Auer, K. \& Al, $E ., 1997$. Free cortisol levels after awakening: a reliable biological marker for the assessment of adrenocortical activity. Life Sciences., 61, 2539-49.

Rayasam, G., Tulasi, V., Sodhi, R., Davis, J. \& Ray, A., 2009. Glycogen synthase kinase 3: more than a namesake. Br J Pharmacol, 156, 885-98.

Razzaghy-Azar, M., Nourbakhsh, M., Pourmoteabed, A., Nourbakhsh, M., llbeigi, D. \& Khosravi, M., 2016. An evaluation of acylated ghrelin and obestatin levels in childhood obesity and their association with insulin resistance, metabolic syndrome, and oxidative stress. J Clin Med, 5.

Reichenberg, A., Yirmiya, R., Schuld, A., Kraus, T., Haack, M., Morag, A. \& Pollmacher, T., 2001. Cytokine Associated Emotional and Cognitive Disturbances in Humans. Arch Gen Psychiatry, 58, 445-452.

Ricken, R., Adli, M., Lange, C., Krusche, E., Stamm, T., Gaus, S., Koehler, S. \& Al, E., 2013. Brain-derived neurotrophic factor serum concentrations in acute depressive patients increase during lithium augmentation of antidepressants. $J$ Clin Psychopharmacol, 33, 806-9.

Ridout, K., Ridout, S., Price, L., Sen, S. \& Tyrka, A., 2016. Depression and Telomere Length: A Meta-Analysis. Journal of affective disorders, 191, 237-247.

Rottenberg, J., 2007. Cardiac vagal control in depression: A critical analysis. Biological Psychology, 74, 200-211.

Ruddick, J., Evans, A., Nutt, D., Lightman, S., Rook, G. \& Lowry, C., 2006. Tryptophan metabolism in the central nervous system: medical implications. Expert Rev Mol Med, 8, 1-27.

Ryan, R., Booth, S., Spathis, A., Mollart, S. \& Clow, A., 2016. Use of salivary diurnal cortisol as an outcome measure in randomised controlled trials: a systematic review. Ann Behav Med, 50, 210-36.

Sapolsky, R.M., 2000. Glucocorticoids and hippocampal atrophy in neuropsychiatric disorders. Archives of General Psychiatry, 57, 925-935.

Sawada, T. \& Yokoi, K., 2010. Effect of zinc supplementation on mood states in young women: a pilot study. Eur J Clin Nutr, 64, 331-3.

Scheffer, M., Bascompte, J., Brock, W.A., Brovkin, V., Carpenter, S.R., Dakos, V., Held, H., Van Nes, E.H., Rietkerk, M. \& Sugihara, G., 2009. Early-warning signals for critical transitions. Nature, 461, 53-59.

Schinder, A. \& Poo, M., 2000. The neurotrophin hypothesis for synaptic plasticity. Trends Neurosci, 23, 639-45.

Schüle, C., Baghai, T., Eser, D., Häfner, S., Born, C., Herrmann, S. \& Rupprecht, R., 2009. The Combined Dexamethasone/CRH Test (DEX/CRH Test) and 
Prediction of Acute Treatment Response in Major Depression. PLoS ONE, 4, e4324.

Seib, C., Whiteside, E., Humphreys, J., Lee, K., Thomas, P., Chopin, L., Crisp, G., O'keeffe, A., Kimlin, M., Stacey, A. \& Anderson, D., 2014. A longitudinal study of the impact of chronic psychological stress on health-related quality of life and clinical biomarkers: protocol for the Australian Healthy Aging of Women Study. BMC Public Health, 14, 9.

Sen, S., Duman, R. \& Sanacora, G., 2008. Serum BDNF, Depression and AntiDepressant Medications: Meta-Analyses and Implications. Biological psychiatry, 64, 527-532.

Siu, A., Bibbins-Domingo, K., Grossman, D., Baumann, L., Davidson, K., Ebell, M., García, F. \& Al., E., 2016. US Preventive Services Task Force Recommendation Statement: Screening for depression in adults. JAMA, 315, 380-7.

Stapelberg, N., Neumann, D., Shum, D., Mcconnell, H. \& Hamilton, C., 2015. From, physiome to pathome: a systems biology model of major depressive disorder and the Psycho-Immune-Neuro-Endocrine network. Current Psychiatry Reviews, 11, 32-62.

Strawbridge, R., Young, A.H. \& Cleare, A.J., 2017. Biomarkers for depression: recent insights, current challenges and future prospects. Neuropsychiatric Disease and Treatment, 13, 1245-1262.

Sviridonova, M.A., Fadeyev, U.V., Sych, Y.P. \& Melnichenko, G.A., 2012. Clinical Significance of TSH Circadian Variability in patients with hypothyroidism. 's.

Tanabe, A. \& Nomura, S., 2007. Pathophysiology of Depression. Nippon Rinsho, 65, $1585-1590$.

Taylor, D., Paton, C. \& Kapur, S., 2015. The Maudsley Prescribing Guidelines, Eleventh Edition. 10 ed.: CRC Press.

Tokunaga, C., Yoshino, K. \& Yonezawa, K., 2004. mTOR integrates amino acid- and energy-sensing pathways. Biochemical and Biophysical Research Communications, 313, 443-446.

Tornatore, L., Thotakura, A.K., Bennett, J., Moretti, M. \& Franzoso, G., 2012. The nuclear factor kappa B signaling pathway: integrating metabolism with inflammation. Trends in Cell Biology, 22, 557-566.

Tortorella, C., Neri, G. \& Nussdorfer, G., 2007. Galanin in the regulation of the hypothalamic-pituitary-adrenal axis (Review). Int J Mol Med, 19, 639-47.

Tsankova, N., Renthal, W., Kumar, A. \& Nestler, E.J., 2007. Epigenetic regulation in psychiatric disorders. Nat Rev Neurosci, 8, 355-67.

Van De Ven, A.C., Muntjewerff, J.W., Netea-Maier, R.T., De Vegt, F., Ross, H.A., Sweep, F.C., Kiemeney, L.A., Vos, P.E., Buitelaar, J.K., Hermus, A.R., Den Heijer, M. \& Janzing, J.G., 2012. Association between thyroid function, thyroid autoimmunity, and state and trait factors of depression. Acta Psychiatr Scand, $126,377-84$.

Varghese, E. \& Brown, E., 2001. The Hypothalamic-Pituitary-Adrenal Axis in Major Depressive Disorder: A Brief Primer for Primary Care Physicians. Primary Care Companion to The Journal of Clinical Psychiatry, 3, 151-155.

Vidal, P.M., Lemmens, E., Dooley, D. \& Hendrix, S., 2012. The role of antiinflammatory cytokines in axon regeneration. Cytokine and Growth Factor Reviews, 24, 1-12.

Vlahopoulos, S.A., Cen, O., Hengen, N., Agan, J., Moschovi, M., Critselis, E., Adamaki, M., Bacopoulou, F., Copland, J.A., Boldogh, I., Karin, M. \& Chrousos, G.P., 2015. Dynamic aberrant NF-кB spurs tumorigenesis: a new 
model encompassing the microenvironment. Cytokine \& growth factor reviews, 26, 389-403.

Weber, B., Schweiger, U., Deuschle, M. \& Heuser, I., 2000. Major depression and impaired glucose tolerance. Exp Clin Endocrinol Diabetes, 108, 187-90.

Wei, J., Sun, G., Zhao, L., Liu, X., Lin, D., Li, T. \& Ma, X., 2014. Hair thyroid hormones concentration in patients with depression changes with disease episodes in female Chinese. Psychiatry Research, 220, 251-253.

Welberg, L., 2010. Psychiatric disorders: Ketamine modifies mood through mTOR. Nat Rev Neurosci, 11, 666-667.

Wichers, M. \& Macs, M., 2002. the Psychoncuroimmuno-patho-physiology of cytokine induced depression in humans. Int $J$ Neuropsychopharmacol, 5, 375-88.

Winokur, A., Maislin, G., Phillips, J. \& Amsterdam, J., 1988. Insulin resistance after oral glucose tolerance testing in patients with major depression. Am J Psychiatry, 145, 325-30.

World Health Organisation, 2009. Global health risks : mortality and burden of disease attributable to selected major risks. Geneva: World Health Organization.

World Health Organization, W.I.-R., 1992. The ICD-10 classification of mental and behavioural disorders: clinical descriptions and diagnostic guidelines. Geneva, World Health Organization.

Young, J., Silber, T., Bruno, D., Galatzer-Levy, I., Pomara, N. \& Marmar, C., 2016. Is there progress? An overview of selecting biomarker candidates for major depressive disorder. Frontiers in Psychiatry, 7.

Zanos, P., Moaddel, R., Morris, P.J., Georgiou, P., Fischell, J., Elmer, G.I., Alkondon, M., Yuan, P., Pribut, H.J., Singh, N.S., Dossou, K.S., Fang, Y., Huang, X.P., Mayo, C.L., Wainer, I.W., Albuquerque, E.X., Thompson, S.M., Thomas, C.J., Zarate, C.A., Jr. \& Gould, T.D., 2016. NMDAR inhibition-independent antidepressant actions of ketamine metabolites. Nature, 533, 481-6.

Zhang, J., Lam, S., Kong, A., Ma, R., Li, S., Chan, J., Yu, M. \& Al., E., 2016. Family conflict and lower morning cortisol in adolescents and adults: modulation of puberty. Sci Rep, 6, 22531.

Zhang, Z.J., Wang, D., Man, S.C., Ng, R., Mcalonan, G.M., Wong, H.K., Wong, W., Lee, J. \& Tan, Q.R., 2014. Platelet 5-HT(1A) receptor correlates with major depressive disorder in drug-free patients. Prog Neuropsychopharmacol Biol Psychiatry, 53, 74-9.

Zheng, J., Skufca, J.D. \& Bollt, E., 2013. Heart rate variability as determinism with jump stochastic paramters. Mathematical biosciences and engineering: $M B E$, $10,1253-1264$.

Zheng, P., Chen, J., Zhou, C., Zeng, L., Li, K., Sun, L., Liu, M. \& Al., E., 2016. Identification of sex-specific urinary biomarkers for major depressive disorder by combined application of NMR- and GC-MS-based metabonomics. Translational Psychiatry, 6, e955.

Zhu, C.B., Blakely, R.D. \& Hewlett, W.A., 2006. The Pro-Inflammatory Cytokines Interleukin One Beta and Tumour Necrosis Factor Alpha Activate Serotonin Transporters. Neuropsychopharmacology, 31, 2121-2131. 
Table 1: Proposed extracellular biomarkers in screening for MDD

\begin{tabular}{|l|l|}
\hline Extracellular Tipping Point Biomarkers \\
\hline Serum unless otherwise stated \\
\hline HPA Dysfunction and Glucocorticoid Resistance \\
\hline Skew & Salivary cortisol on awakening and at 60 minutes \\
\hline \multirow{2}{*}{$\begin{array}{l}\text { Resistance to } \\
\text { change }\end{array}$} & $\begin{array}{l}\text { Salivary cortisol in response to awakening at baseline, 30, 60, 90 } \\
\text { minutes }\end{array}$ \\
\cline { 2 - 2 } & $\begin{array}{l}\text { Nuclear receptor subfamily 3 group C member 1 (NR3C1 or } \\
\text { glucocorticoid receptor) concentration in leucocytes }\end{array}$ \\
\cline { 2 - 2 } & $\begin{array}{l}\text { Combined Dexamethasone Suppression Test (DST) and } \\
\text { Corticoliberin (CRH) stimulation test }\end{array}$ \\
\cline { 2 - 2 } & $\begin{array}{l}\text { Trier Social Stress Test (TSST) stress test using salivary cortisol } \\
\text { concentration and corticotrophin (ACTH) at 10, 30 and 60 } \\
\text { minutes or CO2 stress test using same parameters as TSST }\end{array}$ \\
\hline Flickering & No biomarker identified \\
\hline Increased variance & $\begin{array}{l}\text { 24 hour urine free cortisol or early-morning urine free cortisol to } \\
\text { creatinine ratio }\end{array}$ \\
\hline Autocorrelation & $\begin{array}{l}\text { Repeated methylated nuclear receptor subfamily 3 group C } \\
\text { member 1 (NR3C1 or glucocorticoid receptor) concentration in } \\
\text { leucocytes }\end{array}$ \\
\hline Metabolic Dysfunction \\
\hline Skew & C-reactive protein: cortisol binding globulin (CRP:CBG) ratio \\
\cline { 2 - 2 } & $\begin{array}{l}\text { Anti-thyroperoxidase antibody concentration (anti-TPO } \\
\text { antibodies) }\end{array}$ \\
\cline { 2 - 2 } Ratio of leptin to ghrelin concentrations \\
\hline Resistance to \\
change & $\begin{array}{l}\text { Peak thyroid stimulating hormone (TSH) concentration at 0300 } \\
\text { hours }\end{array}$ \\
\cline { 2 - 2 } CRP:CBG ratio with multiple tests over time \\
\hline Flickering & Heart rate variability \\
\hline Increased variance & Haemoglobin A 1 ic concentration \\
\hline Autocorrelation & Hair free T3 concentration \\
\hline & Repeated Haemoglobin A ic over time \\
\hline
\end{tabular}


Table 2: Proposed intracellular biomarkers in screening for MDD

\begin{tabular}{|c|c|}
\hline \multicolumn{2}{|c|}{ Intracellular Tipping Point Biomarkers } \\
\hline \multicolumn{2}{|c|}{ Serum unless otherwise stated } \\
\hline \multicolumn{2}{|c|}{ Dysfunction of Serotonin and Kynurenine Pathways } \\
\hline \multirow[t]{2}{*}{ Skew } & Ratio of concentration of picolinic acid to kynurenic acid \\
\hline & Ratio of concentration of quinolinic acid to kynurenic acid \\
\hline Resistance to change & 5 hydroxytryptamine $1 \mathrm{~A}\left(5-\mathrm{HT}_{1 \mathrm{~A}}\right)$ receptors in leucocytes \\
\hline Flickering & No biomarker identified \\
\hline \multirow[t]{2}{*}{ Increased variance } & $\begin{array}{l}\text { Ratio of } 24 \text { hour urine picolinic acid to 5-hydroxy indole acetic } \\
\text { acid (5HIAA) }\end{array}$ \\
\hline & $\begin{array}{l}\text { Ratio of } 24 \text { hour urine quinolinic acid to 5-hydroxy indole } \\
\text { acetic acid (5HIAA) }\end{array}$ \\
\hline Autocorrelation & $\begin{array}{l}\text { Tryptophan to kynurenine; tryptophan to quinolinic acid } \\
\text { tryptophan to free serotonin (5-HT) ratios }\end{array}$ \\
\hline \multicolumn{2}{|c|}{ Inflammation and Mitochondrial Oxidative Stress } \\
\hline \multirow[t]{6}{*}{ Skew } & Galanin concentration \\
\hline & Tumour ncerosis fuctor ulphu (TNFu) sonecn \\
\hline & Nuclear factor kappa-B (NF-kB) concentration \\
\hline & $\mathrm{Zn}$ and $\mathrm{Mg}$ concentrations \\
\hline & Brain derived neurotrophic factor (BDNF) \\
\hline & Circular RNAs (circRNA) \\
\hline \multirow[t]{2}{*}{ Resistance to change } & Concurrent telomere length in leucocytes (LTL) \\
\hline & Glycogen synthase kinase 3 (GSK-3) activity in leucocytes \\
\hline Flickering & Heart rate variability \\
\hline \multirow[t]{3}{*}{ Increased variance } & $\begin{array}{l}24 \text { hour urine ratio kappa to lambda light chains for NF-кB or } \\
\text { early-morning kappa }\end{array}$ \\
\hline & light chain to cueatinine ratio in urine for NF- $\mathrm{kB}$ \\
\hline & Concentration of circular RNAs (circRNAs) \\
\hline \multirow[t]{2}{*}{ Autocorrelation } & Mechanistic target of rapamycin (mTOR) activity in leucocytes \\
\hline & Ratio of interleukin 6 to interleukin 10 (IL-6: IL-10) \\
\hline
\end{tabular}

